\title{
Influence of fission modes on prompt neutron characteristics in the neutron-induced fission of ${ }^{235} \mathrm{U}$
}

\author{
P. Talou
}

CEA/DEN/DER/SPRC/Laboratoire d'Études de Physique, Cadarache, France

\begin{abstract}
The de-excitation of the fission fragments formed in the neutron-induced fission of ${ }^{235} \mathrm{U}$, for incident energies from 0.5 to $6.0 \mathrm{MeV}$, is simulated numerically. Neutrons are emitted sequentially from a Weisskopf spectrum with a temperature set by previous neutron emissions. The complete decay chain is followed until a fission product is formed and no further neutron emission is allowed energetically. Detailed results are obtained, such as the average prompt neutron multiplicity as a function of the fragment mass and the total kinetic energy $\bar{v}(\mathrm{~A}, \mathrm{TKE})$ and the multiplicity distribution $\mathrm{P}(v)$. An interpretation in terms of fission modes is proposed.
\end{abstract}

\section{Introduction}

In our understanding of the nuclear fission process, what happens near the scission point where the two nascent fragments separate remains unclear. In particular, how the total excitation energy (TXE) is shared among the light and heavy fragments at scission is still unknown, albeit of great importance. Right after scission, the excited fission fragments will release their intrinsic energies by emitting particles, and most notably the so-called prompt neutrons and gammas. Therefore, studying the characteristics of those particles help to understand the physics near the scission point. From an applied point of view, the characteristics, and in particular the average multiplicity $\bar{v}_{p}$ and emission spectrum $N(\mathrm{E})$ of the prompt neutrons, play a crucial role in nuclear reactor technologies.

Modern libraries of evaluated nuclear data contain information on $\bar{v}_{p}$ and $N(\mathrm{E})$ only. Such data are often obtained by application of the Los Alamos or Madland-Nix model, either in its original form [1] or in a generalized development [2, 3]. However, average quantities are just that, average, and can sometimes hide more profound physics with important implications. Again from the applied point of view, looking beyond averaged quantities can lead to a better agreement with experimental data and to the creation of a more predictive evaluation tool.

Along these lines, recent work [4] focused on following in detail the decay chain initiated from the excited fission fragments. This was done through Monte Carlo simulations of the consecutive emissions of neutrons and gammas from the fission fragments, until the ground-state of a fission product is reached (before eventually undergoing a further beta-decay). Calculations were performed for the neutron induced fission of ${ }^{235} \mathrm{U}$ at $E_{n}=0.5 \mathrm{MeV}$ and for the spontaneous fission of ${ }^{252} \mathrm{Cf}[4]$.

In the present work, the same technique is used to study the neutron-induced fission of ${ }^{235} \mathrm{U}$ for $E_{n}=0.5$ to $6.0 \mathrm{MeV}$, using the experimental data by F.-J. Hambsch [5] for the fission fragments yields Y(A,TKE). These yields were later interpreted by Hambsch in terms of Brosa modes, standards I, II and super-long [6]. Here, we will use this interpretation to look into the characteristics of prompt neutrons for each fission mode. Since a mode corresponds to a particular set of nuclear shapes at the scission point, it is of interest to study its influence on the subsequent emission of prompt neutrons.

This paper is organized as follows: the methodology is first briefly introduced. A presentation and discussion of the numerical results follow. Finally, some ideas are proposed to go further in this type of studies.

\section{The model}

\subsection{Methodology}

The methodology used in this work is discussed only briefly here. For a more detailed presentation, the interested reader is referred to ref. [4].

A Monte Carlo sampling of an initial distribution Y(A, $\mathrm{Z}, \mathrm{TKE})$ is performed to select an initial fragmentation $\left(\mathrm{A}_{l}\right.$, $\left.\mathrm{Z}_{l}, \mathrm{~A}_{h}, \mathrm{Z}_{h}\right)$ and total kinetic energy TKE. The energy balance relation $\mathrm{Q}=\mathrm{TKE}+\mathrm{TXE}$ is then used to obtain the total excitation energy TXE. The total excitation energy TXE available at scission can be written as

$$
T X E=E_{\text {int }}^{*}+E_{L}^{\text {def }}+E_{H}^{\text {def }},
$$

where $E_{i n t}^{*}$ is the intrinsic excitation energy of the compound nucleus, and $E_{L, H}^{d e f}$ are the deformation energies of the light and heavy fragments, at the scission point. In the case of spontaneous and low-energy fission, the intrinsic excitation energy $E_{\text {int }}^{*}$ must be small compared to $E_{i}^{d e f}$. If we neglect its contribution, we are left with the determination of the deformation energies of the two fragments, as given by the difference between the deformation energies at scission and in the ground-state of the fragment. So, even assuming statistical equilibrium at scission, which is not so obvious, the temperatures in light and heavy fragments can still differ. Ohsawa used the parameter [2]

$$
R_{T}=\left\langle T_{L}\right\rangle /\left\langle T_{H}\right\rangle
$$

to include a bias in the original Madland-Nix model which assumed equal values for the temperatures in the light and heavy fragments. In ref. [2], $R_{T}$ is used as an average parameter, independent of the mass split. Obviously, for symmetric 
fission, one would expect $R_{T}=1$, while deviating from this value for asymmetric values. In the present work, $R_{T}$ is used as a parameter, function of the fission mode. Several assumptions were considered and are discussed in the next section.

Once the initial excitation energy of each fragment is known, we follow the decay chain until there is not enough energy left in the system to emit any additional neutron. The probability to emit a neutron of energy $E_{n}$ is governed by the Weisskopf probability distribution at temperature $T$. The emission of this neutron then leads to the compound nucleus $(\mathrm{A}-1, \mathrm{Z})$ at a new temperature characterized by the excitation energy $U_{\text {init }}^{*}-B_{n}(A, Z)-E_{n}$. By looking at this level of details, new quantities can be assessed, e.g., the distribution $\bar{v}(\mathrm{~A}, \mathrm{TKE})$ of the average multiplicity as a function of the fragment mass and total kinetic energy, the multiplicity distribution $\mathrm{P}(v)$, energy and angular correlations among emitted neutrons, etc. We argue that these quantities are invaluable for deepening our understanding of the fission process.

\subsection{Model and input parameters}

The approach described above relies on several input data. In particular, the fission fragment (i.e., pre-neutron emission) yields distribution $\mathrm{Y}(\mathrm{A}, \mathrm{Z}$, TKE) has to be known. While its theoretical prediction remains a challenge, we have used the recent experimental data by F.-J. Hambsch [5] for incident neutron energies from 0.5 up to $6 \mathrm{MeV}$ (just below the onset of second chance fission). The most probable charge $Z_{p}$ of fission fragments is obtained assuming an unchanged charge distribution (UCD). In addition to the raw experimental data, Hambsch also obtained the decomposition of the full distribution into three modes, S1 (standard I), S2 (standard II) and SL (super-long).

Nuclear masses were taken from the tables of Audi, Wapstra and Thibault [7], and level density parameters were taken from ref. [8].

Compound nucleus cross section calculations were performed for the inverse reaction process for each fission fragment, using the Koning-Delaroche global potential [9].

\section{Numerical results}

The experimental yields $\mathrm{Y}(\mathrm{A}, \mathrm{TKE})$ used as input in the present calculations are shown in figure 1 for incident neutrons at $E_{n}=0.5 \mathrm{MeV}$. The unfolding of the total yields in the three fission modes is also shown. Both the S1 and S2 standard modes lead to asymmetric mass distributions and high total kinetic energy (TKE) values, the $\mathrm{S} 1$ mode leading to the highest TKE-values and narrowest mass-distribution. The SL mode is characteristic of a symmetric mass-distribution with lower TKE values. Table 1 summarizes important mean values for the energy release, total kinetic energy and total excitation energy, for each mode. Averaged prompt neutron multiplicity $\bar{v}$ being roughly proportional to TXE, it is already obvious that more neutrons will be emitted in the SL mode than in the S2 and $\mathrm{S} 1$ modes.
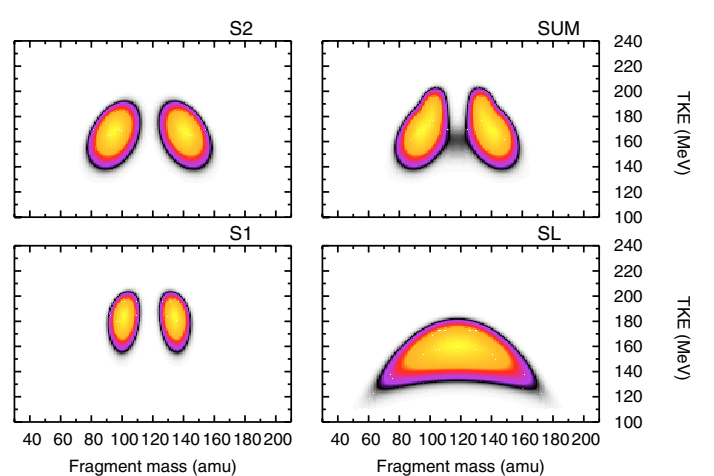

Fig. 1. Experimental yields $\mathrm{Y}(\mathrm{A}, \mathrm{TKE})$ of $\mathrm{n}(0.5 \mathrm{MeV})+{ }^{235} \mathrm{U}$ and their decomposition in fission modes S1, S2 and SL. The scale on the z-axis is different for each plot. The S2 mode largely dominates (74.25\%), with the S1 following (25.53\%) the SL mode contributing very little $(0.22 \%)$ to the full distribution, as can be seen by the shadow in the symmetric mass region in the upper-right plot.

Table 1. Mean values for the energy release $\overline{E_{r}}$, total kinetic energy $\overline{T K E}$, and total excitation energy $\overline{T X E}$ for each fission mode and their weighted sum, and compared to raw experimental data, in the case of $\mathrm{n}(0.5 \mathrm{MeV})+{ }^{235} \mathrm{U}$.

\begin{tabular}{llll}
\hline Mode & $\begin{array}{l}\bar{E}_{r} \\
(\mathrm{MeV})\end{array}$ & $\begin{array}{l}\overline{T K E} \\
(\mathrm{MeV})\end{array}$ & $\begin{array}{l}\overline{T X E} \\
(\mathrm{MeV})\end{array}$ \\
\hline S1 & 192.6 & 182.3 & 17.4 \\
S2 & 185.3 & 167.4 & 24.9 \\
SL & 190.6 & 157.6 & 40.1 \\
Sum & 187.2 & 171.2 & 23.0 \\
\hline Exp. & 187.1 & 171.1 & 23.0 \\
\hline
\end{tabular}

Numerical results for the average neutron multiplicity for the light $\left(\bar{v}_{L}\right)$, heavy $\left(\bar{v}_{H}\right)$, and total $(\bar{v})$ are presented in table 2 . The average neutron energies in the center-of-mass $\left(\bar{E}_{c m}\right)$ and laboratory $\left(\bar{E}_{l a b}\right)$ reference frames are also indicated. The results shown were obtained under different assumptions for the parameter $R_{T}$.

$R_{T}=1.0$ corresponds to the assumption $T_{L}=T_{H}$ at the time of neutron evaporation, thereby neglecting the role played by different deformation energies of the light and heavy fragments. In this case, it is observed that neutrons are emitted preferentially from the heavy fragment, in striking contrast with experimental data, despite the fact that the calculated total average neutron multiplicity $\bar{v}(2.49)$ is in very good agreement with the experimental value (2.47).

The values $R_{T}=1.13$ and 1.34 were taken from ref. [2]. These temperature biases lead to ratios $\bar{v}_{l} / \bar{v}_{h}$ greater than 1 . The best agreement with experimental data is obtained for $R_{T}=1.34$.

In the third set of calculations, $R_{T}$ was chosen as 1.3 for the S1 mode, 1.2 for the S2 mode, and 1.0 for the SL mode. The results for the average neutron multiplicity $\bar{v}_{t o t}, \bar{v}_{l}$ and $\bar{v}_{h}$, are in very good agreement with the experimental data, although the average neutron energies are still underestimated. All following results were obtained under this assumption. 
Table 2. Physical quantities (averaged over all fragment masses) calculated for each mode and their weighted sum.

\begin{tabular}{|c|c|c|c|c|c|}
\hline Mode & $\bar{v}_{L}$ & $\bar{v}_{H}$ & $\bar{v}$ & $\begin{array}{l}\bar{E}_{c m} \\
(\mathrm{MeV})\end{array}$ & $\begin{array}{l}\bar{E}_{l a b} \\
(\mathrm{MeV})\end{array}$ \\
\hline \multicolumn{6}{|l|}{$R_{T}=1.0$} \\
\hline $\mathrm{S} 1$ & 0.93 & 0.66 & 1.59 & 0.82 & 1.63 \\
\hline $\mathrm{S} 2$ & 1.17 & 1.63 & 2.80 & 1.05 & 1.75 \\
\hline SL & 2.31 & 2.11 & 4.43 & 1.30 & 1.98 \\
\hline Sum & 1.11 & 1.38 & 2.49 & 0.99 & 1.72 \\
\hline \multicolumn{6}{|l|}{$R_{T}=1.13$} \\
\hline $\mathrm{S} 1$ & 1.06 & 0.53 & 1.59 & 0.81 & 1.66 \\
\hline $\mathrm{S} 2$ & 1.37 & 1.42 & 2.78 & 1.04 & 1.77 \\
\hline SL & 2.78 & 1.56 & 4.35 & 1.55 & 2.27 \\
\hline Sum & 1.29 & 1.19 & 2.48 & 0.98 & 1.74 \\
\hline \multicolumn{6}{|l|}{$R_{T}=1.34$} \\
\hline $\mathrm{S} 1$ & 1.23 & 0.35 & 1.58 & 0.84 & 1.74 \\
\hline $\mathrm{S} 2$ & 1.64 & 1.12 & 2.76 & 1.05 & 1.84 \\
\hline SL & 2.90 & 1.45 & 4.35 & 1.34 & 2.06 \\
\hline Sum & 1.54 & 0.92 & 2.46 & 1.00 & 1.81 \\
\hline \multicolumn{6}{|c|}{$R_{T}=1.3 / 1.2 / 1.0^{*}$} \\
\hline $\mathrm{S} 1$ & 1.20 & 0.38 & 1.58 & 0.84 & 1.73 \\
\hline $\mathrm{S} 2$ & 1.47 & 1.31 & 2.77 & 1.04 & 1.79 \\
\hline SL & 2.31 & 2.11 & 4.43 & 1.30 & 1.98 \\
\hline Sum & 1.40 & 1.07 & 2.47 & 0.99 & 1.80 \\
\hline \multicolumn{6}{|c|}{ Experimental } \\
\hline Nishio [10] & 1.42 & 1.01 & 2.47 & 1.265 & 2.046 \\
\hline Müller [11] & 1.44 & 1.02 & 2.46 & & \\
\hline
\end{tabular}

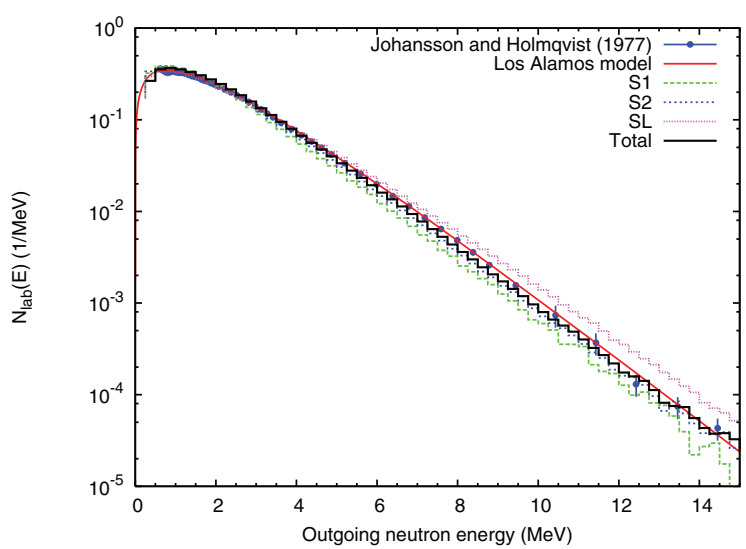

Fig. 2. Prompt neutron spectra in the laboratory frame for each fission mode, at $E_{n}=0.5 \mathrm{MeV}$.

For each fission mode, the calculated prompt neutron spectrum $N(\mathrm{E})$ in the laboratory system is shown in figure 2. The calculated spectrum slightly underestimates the data in the 6-10 MeV energy range.

Figure 3 provides a relatively concise view of the neutron multiplicity as a function of fragment mass and total kinetic energy, for each fission mode. In general, lower values of TKE

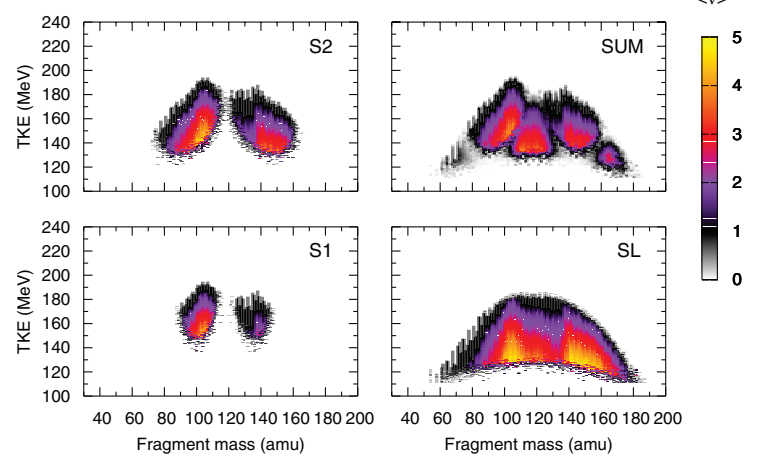

Fig. 3. Average prompt neutron multiplicity as a function of fragment mass and total kinetic energy, for each mode and their weighted sum, at $E_{n}=0.5 \mathrm{MeV}$.

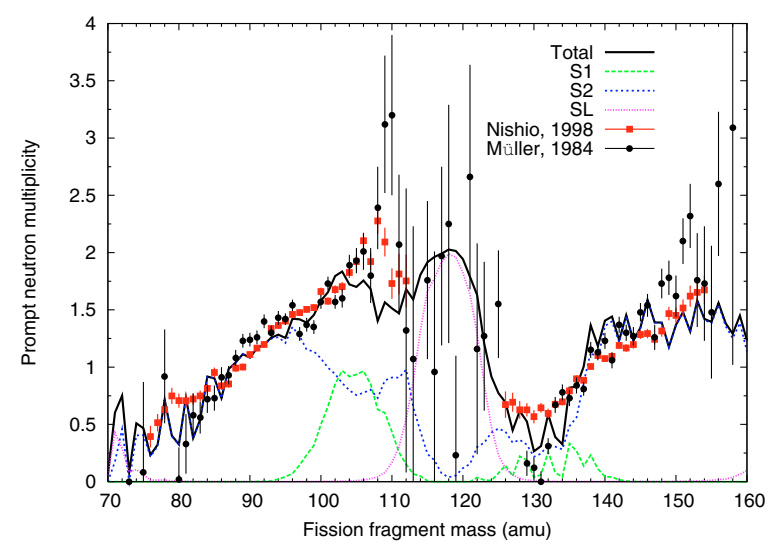

Fig. 4. Average prompt neutron multiplicity as a function of the fragment mass, for each mode and their weighted sum, at $E_{n}=$ $0.5 \mathrm{MeV}$.

lead to higher TXE values, and therefore to a larger number of prompt neutrons. Note that the Q-value of the reaction can influence this simplistic and global interpretation. In the case of the S1 and S2 modes, large values of $R_{T}$ tend to strongly hinder the emission of neutrons from heavy fragments. The asymmetry observed in the SL mode for which $R_{T}=1.0$ can be assigned to different level density parameters in the light and heavy fragments. The total distribution of $\bar{v}(\mathrm{~A}, \mathrm{TKE})$ is shown as "SUM" in this figure. Its specific features have to be understood in terms of dominating fission modes in a particular (A,TKE) region, and are the result of the interplay between the energies TKE, $E_{r}\left(A_{l, h}\right)$ and $E_{l, h}^{d e f}$.

From the two-dimensional distribution $\bar{v}(A, T K E)$ one can infer the distribution $\bar{v}(\mathrm{~A})$, as shown in figure 4 . The wellknown saw-tooth behavior, recognizable in the experimental data by Nishio [10], is also reproduced in our calculations. In the symmetric region, the present calculations lead to higher $\bar{v}$ values since the SL mode dominates there. This result agrees fairly well with data of Müller et al. [11], although statistics are very poor.

Thanks to the level of detail achieved in the present calculations, it is possible to assess the full multiplicity distribution $\mathrm{P}(v)$, and not only its average $\bar{v}$. The result is shown in figure 5 for each fission mode and their weighted sum. Naturally, the 


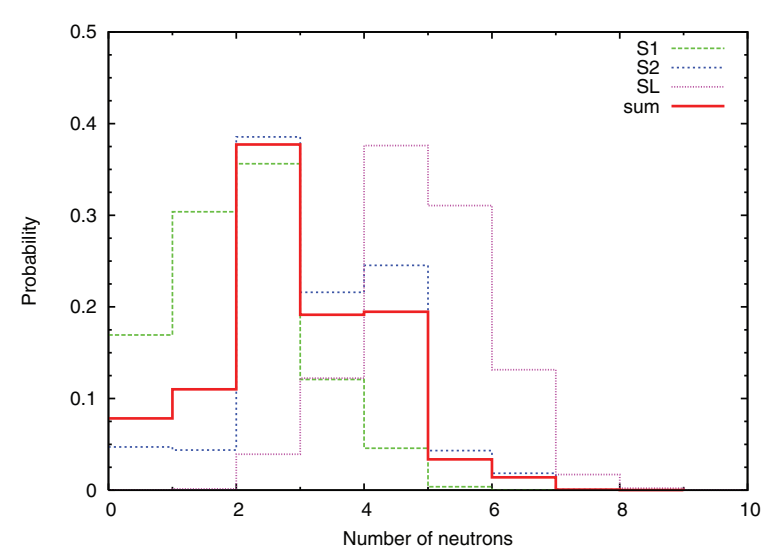

Fig. 5. Prompt neutron multiplicity distribution $\mathrm{P}(v)$ for each fission mode and their weighted distribution, at $E_{n}=0.5 \mathrm{MeV}$.

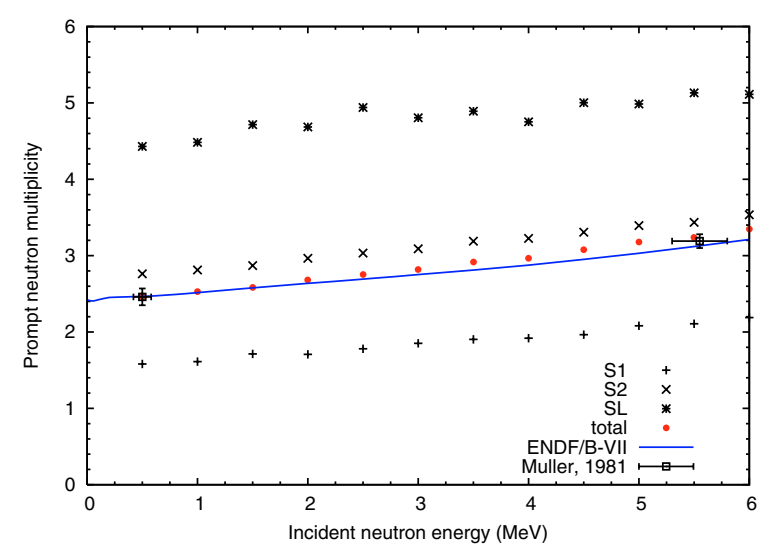

Fig. 6. Prompt neutron multiplicity as a function of the incident neutron energy.

S1 mode is dominant in case of "cold fission" for which $v=0$. On the contrary, the SL mode can lead to large values of $v$. A precise knowledge of $\mathrm{P}(v)$ could be used by experimentalists to calibrate neutron detectors.

It is well known that the asymmetric mass distribution of the fission fragments observed in the case of spontaneous and low-energy fission is due to strong neutron shell effects, and disappear with increasing excitation energies. This is observed in Hambsch's data, where the weight of the symmetric mode increases with the incident neutron energy. The symmetric mode exhibiting a large neutron multiplicity, increasing incident energies lead to an increase in prompt neutron multiplicity more rapidly than if only the S1 and S2 modes were present. The evolution of $\bar{v}$ as a function of the incident neutron energy is shown in figure 6 , as well as its decomposition in fission modes. The present calculations are in very good agreement with the latest ENDF/B-VII evaluation up to $2 \mathrm{MeV}$ incident neutron energy, but overestimate the evaluation above (by up to $4 \%$ at $6 \mathrm{MeV}$ incident energy). However, the present results are consistent with the data of Müller et al. [11] at 5.55 MeV incident energy.

\section{Conclusion}

The study of prompt neutrons is invaluable to learn the physics of the near-scission point. We believe numerical investigations like the one presented here can lead to a better handling of the large body of data stemming from the fission process. However, more work remains to be done on the two (at least) following fronts:

First, we need more (good, obviously) experimental data. However, we cannot be content with only average quantities such as $\bar{v}, \bar{E}$ and $N(\mathrm{E})$. Although those data are of great importance, in particular for existing nuclear applications, they do not tell the full story and do not necessarily lead to a better understanding of the physics at play. On the contrary, information on prompt neutrons and gammas as a function of the fission fragment mass and total kinetic energy are of great importance since they help select between different scenarios of energy partitioning at the scission point.

Second, numerical modeling tools have to be sharpened. The results presented here are very promising and should be improved. The proper Hauser-Feshbach treatment (as opposed to Weisskopf-Ewing) of the evaporation process of the excited fission fragments has to be implemented, in particular to correctly account for the competition between neutron and gamma emission near the neutron binding energy. The sensitivity of the results to the most important parameters (e.g., most probable charge $Z_{p}$ ) should also be studied carefully.

The author is indebted to Dr F.-J. Hambsch for providing his experimental data on fission fragments yields, their interpretation as fission modes "à la Brosa", and his careful reading of the manuscript, and to Dr O. Serot for his always insightful comments on the subject.

\section{References}

1. D.G. Madland, J.R. Nix, Nucl. Sci. Eng. 81, 213 (1982).

2. T. Ohsawa, T. Shibata, in Proceedings of the International Conference on Nuclear Data for Science and Technology, Jülich (1991), p. 965.

3. G. Vladuca, A. Tudora, Ann. Nucl. Energy 28, 419 (2001).

4. S. Lemaire, P. Talou, T. Kawano, D.G. Madland, M.B. Chadwick, Phys. Rev. C 72, 024601 (2005); Phys. Rev. C 73, 014602 (2006).

5. F.-J. Hambsch (2007) (private communication).

6. U. Brosa, S. Grossmann, A. Müller, Phys. Rep. 197(4), 167 (1990).

7. G. Audi, A.H. Wapstra, C. Thibault, Nucl. Phys. A 729, 337 (2003).

8. T. Kawano, S. Chiba, H. Koura, J. Nucl. Sci. \& Tech. 43(1), 1 (2006).

9. A.J. Koning, J.P. Delaroche, Nucl. Phys. A 713, 231 (2003).

10. K. Nishio, Y. Nakagome, H. Yamamoto, I. Kimura, Nucl. Phys. A 632, 540 (1998).

11. R. Müller, A.A. Naqvi, F. Käppeler, F. Dickmann, Phys. Rev. C 29, 885 (1984). 